\title{
Heavy-flavour and quarkonia in heavy-ion collisions
}

\author{
A. Rossi ${ }^{1, a}$ on behalf of ALICE, ATLAS and CMS Collaborations \\ ${ }^{1}$ CERN
}

\begin{abstract}
The comparison of heavy-flavour hadron production in proton-proton, proton- $\mathrm{Pb}$ and $\mathrm{Pb}-\mathrm{Pb}$ collisions at the LHC offers the opportunity to investigate the properties of the high-density colour-deconfined state of strongly-interacting matter (Quark Gluon Plasma, QGP) that is expected to be formed in high-energy collisions of heavy nuclei. A review of the main quarkonium and open heavy-flavour results obtained by the ALICE, ATLAS and CMS experiments is presented.
\end{abstract}

\section{Introduction}

The comparison of quarkonium and open heavy-flavour hadron production in proton-proton, proton- $\mathrm{Pb}$ and $\mathrm{Pb}-\mathrm{Pb}$ collisions at the LHC offers the opportunity to investigate the properties of the high-density colour-deconfined state of strongly-interacting matter (Quark Gluon Plasma, QGP) that is expected to form in high-energy collisions of heavy nuclei [1]. Due to their large mass, charm and beauty quarks are created at the initial stage of the collision in hard-scattering processes with high virtuality $\left(Q^{2} \gtrsim 4 \mathrm{~m}_{c[b]}^{2}\right)$ involving partons of the incident nuclei. They interact with the medium and lose energy via both inelastic (medium-induced gluon radiation, or radiative energy loss) [2, 3] and elastic (collisional energy loss) [4] processes. The loss of energy, sensitive to the medium energy density and size, is expected to be smaller for heavy quarks than for light quarks and gluons, due to the smaller colour coupling factor of quarks with respect to gluons, and to the 'dead-cone effect', which reduces small-angle gluon radiation for heavy quarks with moderate energyover-mass values [5]. A sensitive observable is the nuclear modification factor, defined as $R_{\mathrm{AA}}\left(p_{\mathrm{T}}\right)=\frac{\mathrm{d} N_{\mathrm{AA}} / \mathrm{d} p_{\mathrm{T}}}{\left\langle T_{\mathrm{AA}}\right\rangle \times \mathrm{d} \sigma_{\mathrm{pp}} / \mathrm{d} p_{\mathrm{T}}}$, where $N_{\mathrm{AA}}$ is the yield measured in heavy-ion collisions, $\left\langle T_{\mathrm{AA}}\right\rangle$ is the average nuclear overlap function calculated with the Glauber model [7] in the considered centrality range, and $\sigma_{\mathrm{pp}}$ is the production cross section in pp collisions. A similar observable is the central-to-peripheral ratio $\left(R_{\mathrm{CP}}\right)$, in which the yield measured in central collisions is compared to that measured in peripheral ones scaled by the central-to-peripheral ratio of the number of nucleon-nucleon binary collisions estimated with the Glauber model. In-medium energy loss determines a suppression, $R_{\mathrm{AA}}<1$, of hadrons at moderate-to-high transverse momentum $\left(p_{\mathrm{T}} \gtrsim 2 \mathrm{GeV} / c\right)$. The dependence of the energy loss on the parton nature (quark/gluon) and mass can be investigated by comparing the nuclear

\footnotetext{
a e-mail: andrea.rossi@cern.ch
}

modification factors of hadrons with charm $\left(R_{\mathrm{AA}}^{\mathrm{D}}\right)$ and beauty $\left(R_{\mathrm{AA}}^{\mathrm{B}}\right)$ with that of pions $\left(R_{\mathrm{AA}}^{\pi}\right)$, mostly originating from gluon fragmentation. A mass ordering pattern $R_{\mathrm{AA}}^{\pi}\left(p_{\mathrm{T}}\right)<R_{\mathrm{AA}}^{\mathrm{D}}\left(p_{\mathrm{T}}\right)<R_{\mathrm{AA}}^{\mathrm{B}}\left(p_{\mathrm{T}}\right)$ has been predicted [5, 6]. In heavy-ion collisions with non-zero impact parameter the interaction region exhibits an azimuthal anisotropy with respect to the reaction plane $\left(\Psi_{\mathrm{RP}}\right)$ defined by the impact parameter and the beam direction. Collective effects convert this geometrical anisotropy into an anisotropy in momentum space that is reflected in the final hadron azimuthal distribution [8]. The effect, sensitive to the degree of thermalization of the system, can be evaluated by measuring the $2^{\text {nd }}$ coefficient of the Fourier expansion of the azimuthal distribution, called elliptic flow $\left(v_{2}\right)$. The measurement of heavy-flavour particle $v_{2}$ can provide, at low $p_{\mathrm{T}}$, fundamental information on the degree of thermalization of heavy quarks in the medium. At high $p_{\mathrm{T}}$, a non-zero $v_{2}$ can originate from the path-length dependence of energy loss [9-14].

Already in 1986, it was suggested that the formation of $\mathrm{J} / \psi$ could be suppressed in a deconfined medium with free colour charges screening, in a Debye-like way, the QCD $Q \bar{Q}$ potential [15]. The screening should be more effective in "melting" less tightly bound states, leading to the expectation of a sequential suppression pattern of charmonium and bottomonium states, with the higher states melting at smaller Debye temperatures with respect to the $\mathrm{J} / \psi$ and $\Upsilon(1 \mathrm{~S})$ ground states [16]. The observation of $\mathrm{J} / \psi$ suppression in $\mathrm{Pb}-\mathrm{Pb}$ collisions at SPS [17] was interpreted as a signature of deconfinement. However, a similar suppression was observed in $\mathrm{Au}-\mathrm{Au}$ collisions at $\sqrt{s_{\mathrm{NN}}}=200 \mathrm{GeV}$ at RHIC [18], in contrast with the expectation for a higher suppression in the hotter, denser medium produced at RHIC higher colliding energies. The two results might be reconciled by an additional production of $\mathrm{J} / \psi$ arising from coalescence of $\mathrm{c}$ and $\overline{\mathrm{c}}$ quarks in the medium. This $\mathrm{J} / \psi$ regeneration mechanism 
was first proposed by the Statistical Hadronization Model, which assumes deconfinement and thermal equilibrium of the bulk of $c \bar{c}$ pairs to produce $\mathrm{J} / \psi$ at the phase boundary by statistical hadronization only [19]. Later, the transport models proposed a dynamical competition between the $\mathrm{J} / \psi$ suppression by the QGP and the regeneration mechanism, which enables them to describe also the $p_{\mathrm{T}}$ dependence of the $\mathrm{J} / \psi R_{\mathrm{AA}}[21,22]$. These models have in common the assumption of deconfinement and some degree of charm quark thermalization. At the LHC both effects are expected to intensify: a stronger suppression due to the higher energy density and temperature and a larger charmonium yield from recombination due to the more copious production of charm quarks at LHC energies than at SPS/RHIC energies.

The measurement of nuclear effects requires the understanding of the production cross-sections of open heavy-flavour and quarkonia in pp collisions, used as a reference. Open heavy-flavour production is well described by pQCD calculations, like FONLL [24], relying on the factorization approach. For quarkonium, the validity of a factorization approach is not trivial, due to the interplay of perturbative and non-perturbative effects, the latter related to the formation of the non-relativistic $Q \bar{Q}$ bound state. For a review of the heavy-flavour pp results at the LHC and of their theoretical modelling see [25-27]. A $R_{\mathrm{AA}}$ value different from unity can originate also from initial and final state "cold-nuclear matter" effects, not related to the formation of a deconfined medium. At LHC energies, nuclear shadowing, which reduces the parton distribution functions for partons with nucleon momentum fraction $x$ below $10^{-2}$, is expected to be the most important for heavy-flavour production. Coherent parton energy loss in cold nuclear matter and nuclear absorption of the "pre-hadron" $Q \bar{Q}$ pair before hadronization are also expected to affect quarkonium production [23]. A correct interpretation of heavy-ion results demands for the measurement of these effects via the analysis of $\mathrm{p}-\mathrm{Pb}$ data.

In these proceedings, a review of the main quarkonium and open heavy-flavour results obtained by the ALICE, ATLAS and CMS experiments is presented. The focus is on the measurements performed in $\mathrm{Pb}-\mathrm{Pb}$ collisions. The preliminary results on $\mathrm{J} / \psi$ production in $\mathrm{p}-\mathrm{Pb}$ collisions will be discussed as well.

\section{Quarkonia}

ALICE, ATLAS and CMS measured quarkonium production in $\mathrm{Pb}-\mathrm{Pb}$ collisions by reconstructing charmonia and bottomonia states in the di-muon decay channel. At mid-rapidity, ALICE measured $\mathrm{J} / \psi$ production in the di-electron channel. Exploiting the nice complementarity of the kinematic ranges covered by the 3 experiments, quarkonium production is scanned through 4 rapidity units from $p_{\mathrm{T}}=0$ to high $p_{\mathrm{T}}$.

The nuclear modification factor of inclusive $\mathrm{J} / \psi$ for $p_{\mathrm{T}}>0$ in $2.5<y<4$ measured by ALICE as a function of the collision centrality, expressed in terms of number of nucleons participating to the collisions [28], is compared in the top left panel of figure 1 with the measurements performed by the PHENIX experiment in $\mathrm{Au}-\mathrm{Au}$ collisions at $\sqrt{s_{\mathrm{NN}}}=200 \mathrm{GeV}$ at RHIC in $|y|<0.35$ and in $1.2<|y|<2.2$. At the LHC the suppression is independent from the collision centrality for $\left\langle N_{\text {part }}\right\rangle>100$ and a smaller suppression is observed for most central collisions with respect to RHIC. Both transport models [21, 22, 29] and the statistical model $[19,20]$, including a relevant contribution from recombination, can reproduce the data reasonably well $[28,30]$. The same considerations hold at mid-rapidity, where $\mathrm{J} / \psi$ production for $p_{\mathrm{T}}>0$ was measured by ALICE in the di-electron channel in $|y|<0.9$ [30]. Within the rapidity range of the reported measurement, $R_{\mathrm{AA}}$ decreases by about $40 \%$ towards forward rapidity. As a function of transverse momentum, $R_{\mathrm{AA}}$ decreases significantly in central collisions, from about 0.65 at $p_{\mathrm{T}} \sim 0.5 \mathrm{GeV} / c$ to about 0.2 at $p_{\mathrm{T}} \sim 7 \mathrm{GeV} / c$, as shown in the bottom-left panel of figure 1 . The trend is well reproduced by the transport model by Zhao et al. [21], which foresees a substantial contribution from regenerated $\mathrm{J} / \psi$ at low $p_{\mathrm{T}}$, vanishing towards higher $p_{\mathrm{T}}$. For $p_{\mathrm{T}} \gtrsim 6 \mathrm{GeV} / c$, where coalescence should play a minor, if not negligible, role, the suppression, measured by ALICE and ATLAS [31] for inclusive $\mathrm{J} / \psi$ (including $\mathrm{J} / \psi$ from b-hadron decays) and by CMS for prompt only $\mathrm{J} / \psi[32,33]$, increases towards most central collisions (top-right panel of figure 1), does not show a significant dependence on $p_{\mathrm{T}}$ and is independent from rapidity. Though the different trends observed at low $p_{\mathrm{T}}$ and high $p_{\mathrm{T}}$ are in agreement with the expectations from a scenario in which Debye screening is overcome by $\mathrm{J} / \psi$ production from coalescence at low $p_{\mathrm{T}}$, the measurement of $R_{\mathrm{AA}}$ of higher charmonia states, of $\mathrm{J} / \psi \mathrm{v}_{2}$ and of cold nuclear matter effects is required for a conclusive interpretation of the results. Regarding the latter, figure 1 shows on the bottom-right panel the first preliminary measurement of $\mathrm{J} / \psi$ nuclear modification factor in $\mathrm{p}-\mathrm{Pb}$ collisions $\left(R_{\mathrm{pPb}}\right)$, reported by ALICE. In the figure, negative (positive) values of rapidity refer to the case in which the $\mathrm{Pb}(\mathrm{p})$ beam moves towards the ALICE muon spectrometer used for the measurement. The EPS09 NLO shadowing calculations [34, 35] and models including coherent parton energy loss [36] can reproduce the data, while the specific Colour Glass Condensate model described in [37] underestimates the measurement. Calculations based on shadowing EPS09 are not able to reproduce the $\mathrm{Pb}-\mathrm{Pb}$ measurement [28], indicating that the suppression observed in this latter system is a hot medium effect. $\mathrm{J} / \psi$ coming from $c \bar{c}$ recombination inherit the azimuthal anisotropy of charm quarks and, therefore, should show a non-zero $v_{2}$, if charm quarks take part in the collective expansion of the system. A hint of this was observed by ALICE [38], but the statistical uncertainties are still too large and prevent a conclusion. Concerning heavier charmonia states, a preliminary hint for a double ratio of $\psi(2 S)$ to $\mathrm{J} / \psi$ in $\mathrm{Pb}-\mathrm{Pb}$ and pp larger than unity was released by CMS [39] for $3<p_{\mathrm{T}}<30 \mathrm{GeV} / c$ and $1.6<|y|<2.4$, 

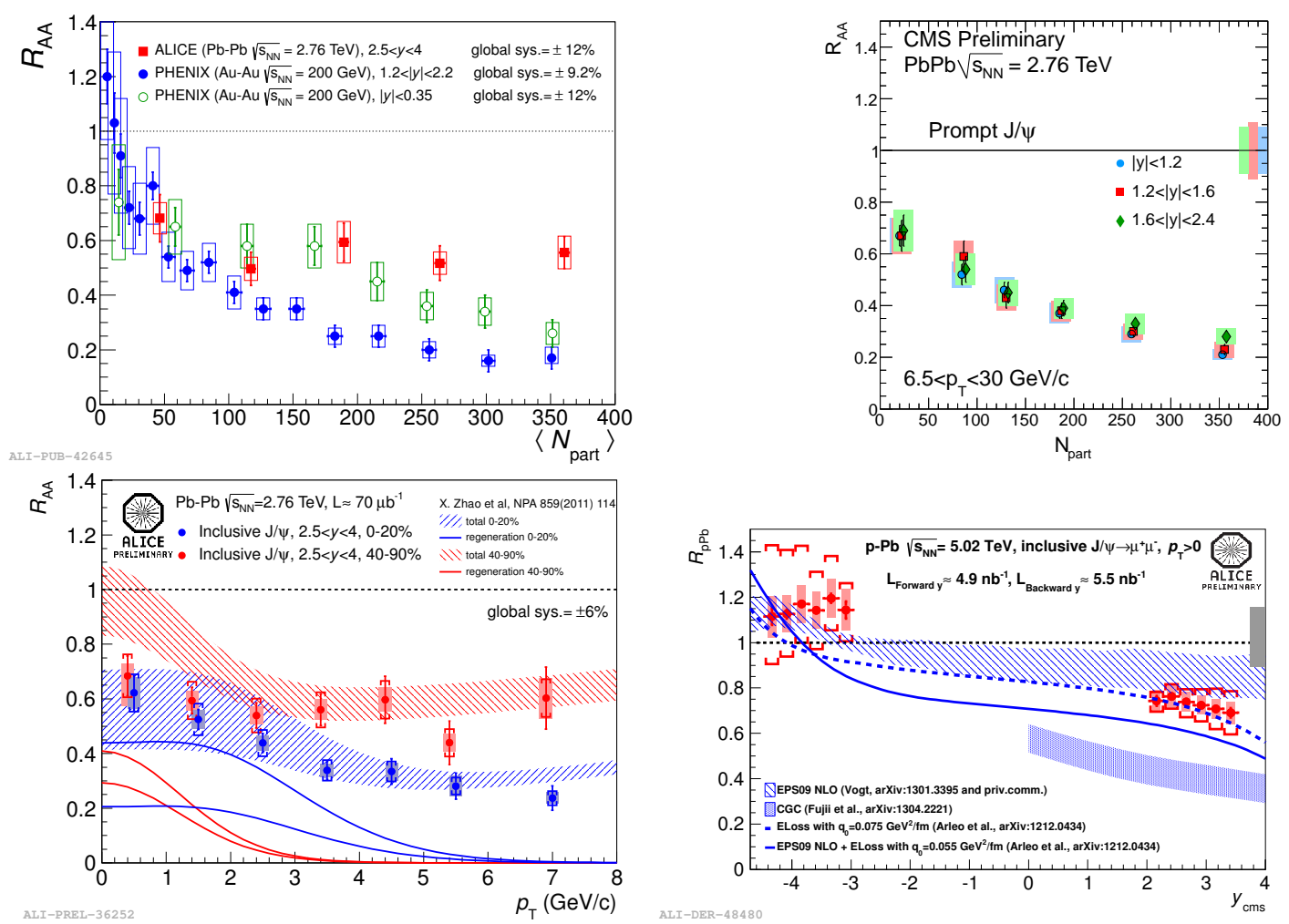

Figure 1. Top: centrality dependence of $\mathrm{J} / \psi R_{\mathrm{AA}}$ for $p_{\mathrm{T}}>0$ in $2.5<y<4$ (left, inclusive $\mathrm{J} / \psi$, figure from [28]) and for $6.5<p_{\mathrm{T}}<30 \mathrm{GeV} / c$ in different rapidity intervals (right, prompt $\mathrm{J} / \psi$, figure from [33]) measured by ALICE and CMS respectively in Pb-Pb collisions at $\sqrt{s_{\mathrm{NN}}}=2.76 \mathrm{TeV}$ at the LHC. ALICE data are compared to the measurements performed by PHENIX in Au-Au collisions at $\sqrt{s_{\mathrm{NN}}}=200 \mathrm{GeV}$ at RHIC. Bottom left: $p_{\mathrm{T}}$ dependence of $\mathrm{J} / \psi R_{\mathrm{AA}}$ compared to the transport model by Zhao et al. [21]. Bottom right: $\mathrm{J} / \psi R_{\mathrm{AA}}$ in $\mathrm{p}-\mathrm{Pb}$ collisions at $\sqrt{s_{\mathrm{NN}}}=5.02 \mathrm{TeV}$ compared to models (see text).

contrasting the expectation of a higher suppression for higher states. The measurement is however affected by large uncertainties, mainly related to the pp reference, and the preliminary measurement by ALICE [40] for $p_{\mathrm{T}}>3 \mathrm{GeV} / c$ and $2.5<y<4$ does not substantiate it. At the moment, there is no measurement of $\chi_{c} R_{\mathrm{AA}}$ available.

The much smaller abundance of bottom quarks with respect to charm quarks should contain the potential contribution of coalescence to bottomonia production to a negligible level. The $\Upsilon(1 \mathrm{~S}) R_{\mathrm{AA}}$ shown in the top panel of figure 2, measured for $p_{\mathrm{T}}>0$ by CMS at mid-rapidity $(|y|<2.4)$ and by ALICE at forward rapidity $(2.5<y<4)$, decreases from about 1 in peripheral events to about 0.4 in central events. No significant dependence on rapidity can be appreciated within the statistical and systematic uncertainties [30]. The observed $R_{\mathrm{AA}}$ is influenced by the suppression level of higher states, whose decay yields $\sim 50 \%$ of the $\Upsilon(1 \mathrm{~S})$ production in pp collisions at high $p_{\mathrm{T}}$. CMS measured the centrality dependence of $\Upsilon(2 \mathrm{~S})$ $R_{\mathrm{AA}}$, which is compared, in the bottom panel of figure 2 , to the ground state $R_{\mathrm{AA}}$ [41]. $R_{\mathrm{AA}}$ goes from about 0.3 in peripheral collisions to $\lesssim 0.1$ in central collisions. Thus, a much stronger suppression is observed with respect to the ground state, in qualitative agreement with the expectation of a sequentially increasing suppression of higher quarkonium states, less tightly bound and with a smaller
Debye temperature. This scenario is also supported by the data reported by CMS in [41] on the $\Upsilon(1 S), \Upsilon(2 S)$ and $\Upsilon(3 \mathrm{~S}) R_{\mathrm{AA}}$ in minimum bias collisions, which also suggest that the direct $\Upsilon(1 \mathrm{~S})$ is almost unsuppressed.

\section{Open heavy-flavour}

Several observables were used to address open charm and beauty production in $\mathrm{Pb}-\mathrm{Pb}$ collisions: the production of prompt D mesons from the full reconstruction of hadronic decay channels at mid-rapidity was measured by ALICE [42, 43], leptons from semi-leptonic decays of heavy-flavour particles were studied by ALICE (electrons in $|\eta|<0.6$ [44], muons in $2.5<y<4$ [45]) and by ATLAS (muons in $|\eta|<1.05$ [46]), while the production of secondary $\mathrm{J} / \psi$ from B decay was measured by CMS [33]. Figure 3 shows the $p_{\mathrm{T}}$ dependence of the average of $\mathrm{D}^{0}$, $\mathrm{D}^{+}$and $\mathrm{D}^{*+}$ meson $R_{\mathrm{AA}}$ in $1<p_{\mathrm{T}}<36 \mathrm{GeV} / c$ in central $0-7.5 \%$ collisions: a strong suppression is observed, by about a factor 5 around $10 \mathrm{GeV} / c$, with a relevant dependence on $p_{\mathrm{T}}$. Within the statistical and systematic uncertainties, the $\mathrm{D}$ meson $R_{\mathrm{AA}}$ is compatible with that of charged hadrons [43]. The ongoing analysis of data from the $\mathrm{p}-\mathrm{Pb}$ run will clarify how much of the observed suppression can be ascribed to a medium effect. However, the main cold nuclear matter effect is expected to be 

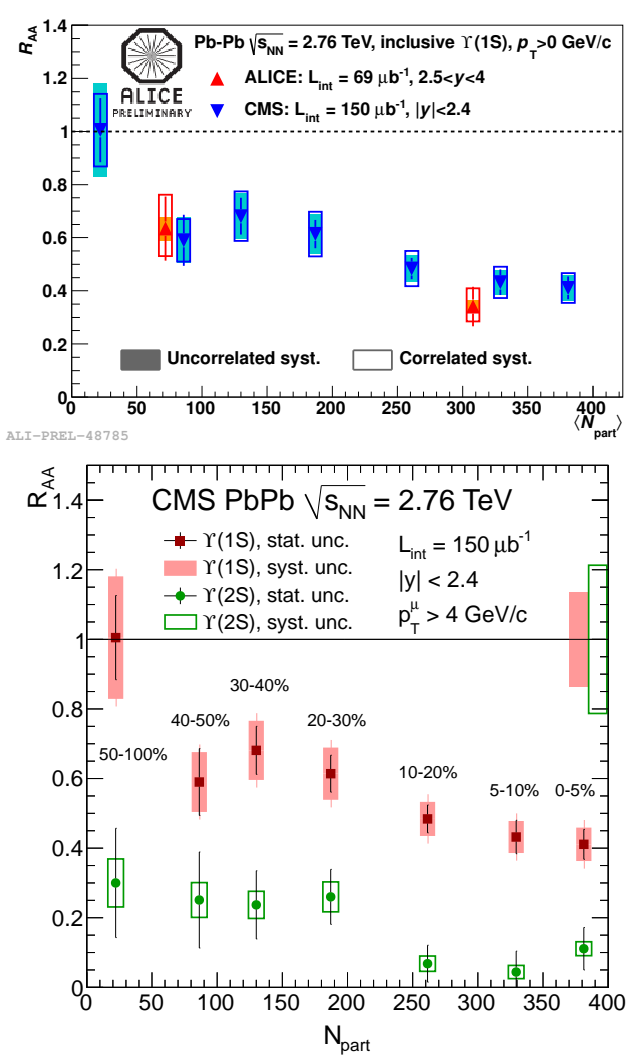

Figure 2. Top: $\Upsilon(1 \mathrm{~S}) R_{\mathrm{AA}}$ measured by ALICE in $2.5<y<4$ [30] and by CMS in $|y|<2.4$ [41] as a function of centrality. Bottom: comparison of $\Upsilon(1 \mathrm{~S})$ and $\Upsilon(2 \mathrm{~S}) R_{\mathrm{AA}}$ measured by CMS [41].

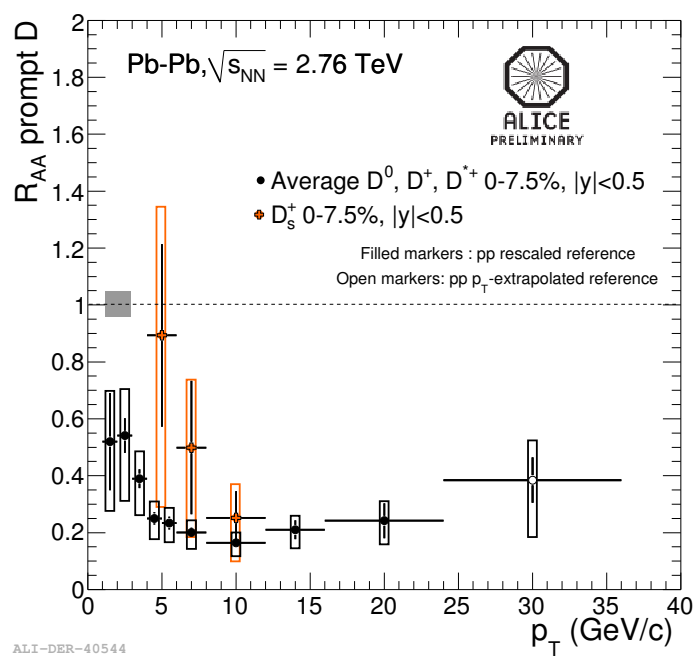

Figure 3. Average of $\mathrm{D}^{0}, \mathrm{D}^{+}$and $\mathrm{D}^{*+} R_{\mathrm{AA}}$ and $\mathrm{D}_{\mathrm{s}} R_{\mathrm{AA}}$ as a function of $p_{\mathrm{T}}$, measured in $0-7.5 \%$ central $\mathrm{Pb}-\mathrm{Pb}$ collisions by ALICE [43, 47].

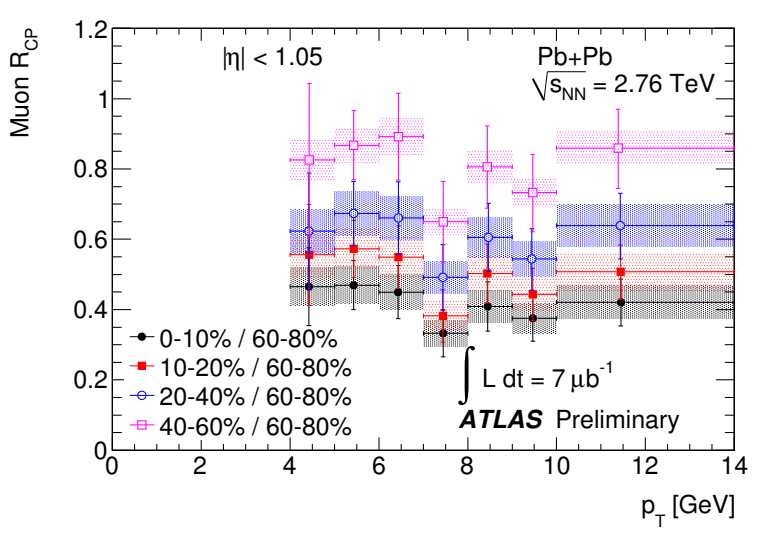

Figure 4. Central-to-peripheral ratio $\left(R_{\mathrm{CP}}\right)$ of muons from heavyflavour hadron decay as a function of $p_{\mathrm{T}}$ in different centralities, measured by ATLAS [46].

nuclear shadowing, which should yield a relatively small effect for $p_{\mathrm{T}} \gtrsim 5 \mathrm{GeV} / c$ [42]. Thus, the observed suppression can be considered an evidence of in-medium charm quark energy loss. In the same figure, the result of the first measurement of the production of the charmed and strange $D_{s}$ meson in heavy-ion collisions is reported [47]. A suppression similar to that of non-strange $\mathrm{D}$ mesons is measured in $8<p_{\mathrm{T}}<12 \mathrm{GeV} / c$. At lower $p_{\mathrm{T}}$, the $\mathrm{D}_{\mathrm{s}}$ $R_{\mathrm{AA}}$ is compatible with that of the non-strange mesons within the large statistical and systematic uncertainties, but there is an intriguing hint for a smaller suppression of $\mathrm{D}_{\mathrm{s}}$ production. A more precise measurement, achievable with larger data samples from future LHC runs, might pose quantitative implications for models which include hadronization via coalescence and foresee an enhancement of $D_{s}$ production deriving from the larger yield of strange quarks in a deconfined medium [48, 49]. In figure 4 , the central-to-peripheral ratio $\left(R_{\mathrm{CP}}\right)$ for muons from heavy-flavour hadron decay measured by ATLAS at mid-rapidity for $p_{\mathrm{T}}>4 \mathrm{GeV} / c$ in different centrality classes is shown. A clear suppression, increasing with centrality, is observed, without any appreciable dependence on transverse momentum. In central collisions, a similar suppression was observed by ALICE for electrons (figure 6, bottom left) and muons [45] from heavy-flavour hadron decay, at central and forward rapidity respectively. In pp collisions, according to FONLL predictions [24], the yield of electrons (muons) from beauty hadron decays exceeds that of electrons (muons) from charm hadron decays for $p_{\mathrm{T}} \gtrsim 5 \mathrm{GeV} / c$. Therefore, the measured $R_{\mathrm{AA}}$ values indicate that the production of beauty hadrons at high $p_{\mathrm{T}}$ is also suppressed in central heavy-ion collisions. A direct evidence of this comes from the measurement of secondary $\mathrm{J} / \psi$ from beauty-hadron decays performed by CMS [33]: $R_{\mathrm{AA}}$ decreases from about 0.8 in peripheral collisions to about 0.4 in central collisions, as shown in figure 5 for $\mathrm{J} / \psi$ in $|y|<1.2$ and $6.5<p_{\mathrm{T}}<30 \mathrm{GeV} / c$. The comparison with the centrality dependence of $\mathrm{D}$ meson $R_{\mathrm{AA}}$, displayed in the same figure, highlights a first indication for the mass dependence of in-medium 


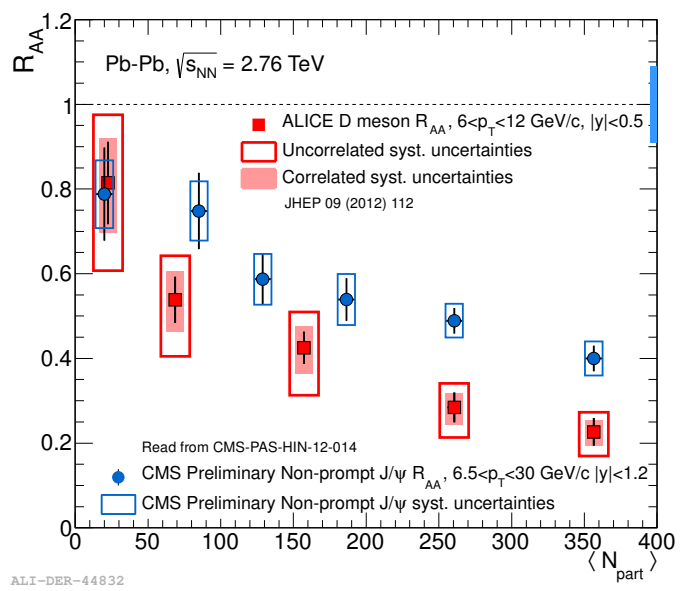

Figure 5. Comparison of the centrality dependence of the $R_{\mathrm{AA}}$ of $\mathrm{D}$ mesons in $6<p_{\mathrm{T}}<12 \mathrm{GeV} / c$ and secondary $\mathrm{J} / \psi$ from bhadron decays in $6.5<p_{\mathrm{T}}<30 \mathrm{GeV} / c$, measured by ALICE [42] and CMS [33] respectively.

partonic energy loss, with $R_{\mathrm{AA}}^{\mathrm{B}}>R_{\mathrm{AA}}^{\mathrm{D}}$. However, a firm and quantitative conclusion is prevented by the fact that the kinematic ranges of the measurements, if considered with respect to the parent quarks, are not the same.

In the top right panel figure 6 the first measurement of D meson elliptic flow in heavy-ion collisions is shown. The measurement, performed by ALICE in the 30-50\% centrality range [50], exploits the event plane method, in which the correlation of the particle azimuthal angle $(\phi)$ to the reaction plane $\Psi_{\mathrm{RP}}$ is analyzed. The reaction plane is estimated via the event plane $\Psi_{2}$, by the so-called $Q_{2}$ vector, which is obtained from the azimuthal distribution of a (sub-)sample of tracks in the event [51]. Although the uncertainties are sizable, a significantly non-zero $v_{2}$ is observed in $2<p_{\mathrm{T}}<6 \mathrm{GeV} / c$, with an average of the measured values in this range around 0.2. A positive $v_{2}$ is also observed for $p_{\mathrm{T}}>6 \mathrm{GeV} / c$, which most likely originates from the path-length dependence of the partonic energy loss, although the large uncertainties do not allow a firm conclusion. The measured D meson $v_{2}$ is comparable in magnitude to that of charged particles, which is dominated by light-flavour hadrons [52]. This suggests that low momentum charm quarks take part in the collective motion of the system. A further indication in this direction is provided by the $v_{2}$ of electrons from heavy-flavour decay (same figure, bottom right panel), measured by ALICE with the event plane method in the $20-40 \%$ centrality range. The measured D meson and electron $v_{2}$ tend to favour models like BAMPS [9], UrQMD [10] and Coll+LPM radiative [11] that predict a larger anisotropy at low $p_{\mathrm{T}}$. However, as shown on the left of the same figure, the same models have difficulties in reproducing also the $\mathrm{D}$ meson and heavy-flavour electron nuclear modification factors in the whole measurement momentum ranges, whereas models like POWLANG [12], WHDG rad+coll [13] and TAMU (Rapp et al.) [14] are more challenged by the description of heavy-flavour elliptic flow.

\section{Conclusions}

The smaller $\mathrm{J} / \psi$ suppression measured in central heavyion collisions at LHC with respect to RHIC and the observed centrality and $p_{\mathrm{T}}$ trends favour a scenario with a significant contribution to $\mathrm{J} / \psi$ production at low $p_{\mathrm{T}}$ from recombination of deconfined $c$ quarks, vanishing at higher $p_{\mathrm{T}}$ where Debye screening determines the production features. The observation of a larger suppression of $\Upsilon(2 S)$ and $\Upsilon(3 S)$ than $\Upsilon(1 S)$ supports the Debye-screening picture of a sequential suppression of states following the hierarchy of binding energies. However, any firm conclusion is still prevented by the lack of information on the suppression of higher states, where, especially in the charmonia case, the measurements are characterized by a poor precision (for $\psi(2 s)$ ) or are absent (for $\chi_{c}$ and $\chi_{b}$ ), by the theoretical prediction uncertainties, mostly dominated by the uncertainty on the total charm production cross-section in $\mathrm{Pb}-\mathrm{Pb}$ collisions, and by the uncertainties related to coldnuclear matter effects, the nature of which should be clarified by the ongoing analysis of $\mathrm{p}-\mathrm{Pb}$ data. More data from future runs at the LHC should allow for a more precise measurement of $\mathrm{J} / \psi v_{2}$, that, beside addressing recombination, could also help to assess the degree of charm quark thermalization in the medium. An indication of charm thermalization is provided by the measurement of a nonzero $\mathrm{D}$ meson $v_{2}$ at low $p_{\mathrm{T}}$, with values similar to those of charged hadron $v_{2}$. Despite the large uncertainties, this observation complements the measurement of a large suppression of $\mathrm{D}$ meson production at high $p_{\mathrm{T}}$ (about a factor 5 at $p_{\mathrm{T}} \sim 10 \mathrm{GeV} / c$ ) in central $\mathrm{Pb}-\mathrm{Pb}$ collisions, which constitutes an evidence of in-medium charm energy loss and points to a strong interaction of charm with the QGP. The comparison of the $R_{\mathrm{AA}}$ of $\mathrm{D}$ mesons and of secondary $\mathrm{J} / \psi$ from b-hadron decays suggests that beauty loses less energy than charm, corroborating the prediction of a dependence of energy loss from the quark mass induced by the dead-cone effect. However, a satisfactory description of the interaction of charm and beauty quarks with the medium is still missing as highlighted by the absence of a model able to reproduce simultaneously the $R_{\mathrm{AA}}$ and $v_{2}$ of D mesons and electrons from heavy-flavour hadron decay. Although the results obtained at the LHC after the first 3 years of data taking can be casted into a qualitatively understood frame, more precise and "differential" measurements are needed to focus the emerging picture of heavy-flavour production and interaction in the QGP. The data collected in the next runs and, even more, the upgrade of the detectors, during the long shut down in 2018, should allow to reach this target and bring experimental heavy-ion physics into the charm and beauty era of QGP.

\section{References}

[1] P. Braun-Munzinger and J. Stachel, Nature 448, 302309 (2007).

[2] M. Gyulassy and M. Plumer, Phys. Lett. B243, 432 (1990).

[3] R. Baier, Y. L. Dokshitzer, A. H. Mueller, S. Peigne and D. Schiff, Nucl. Phys. B484, 265 (1997). 

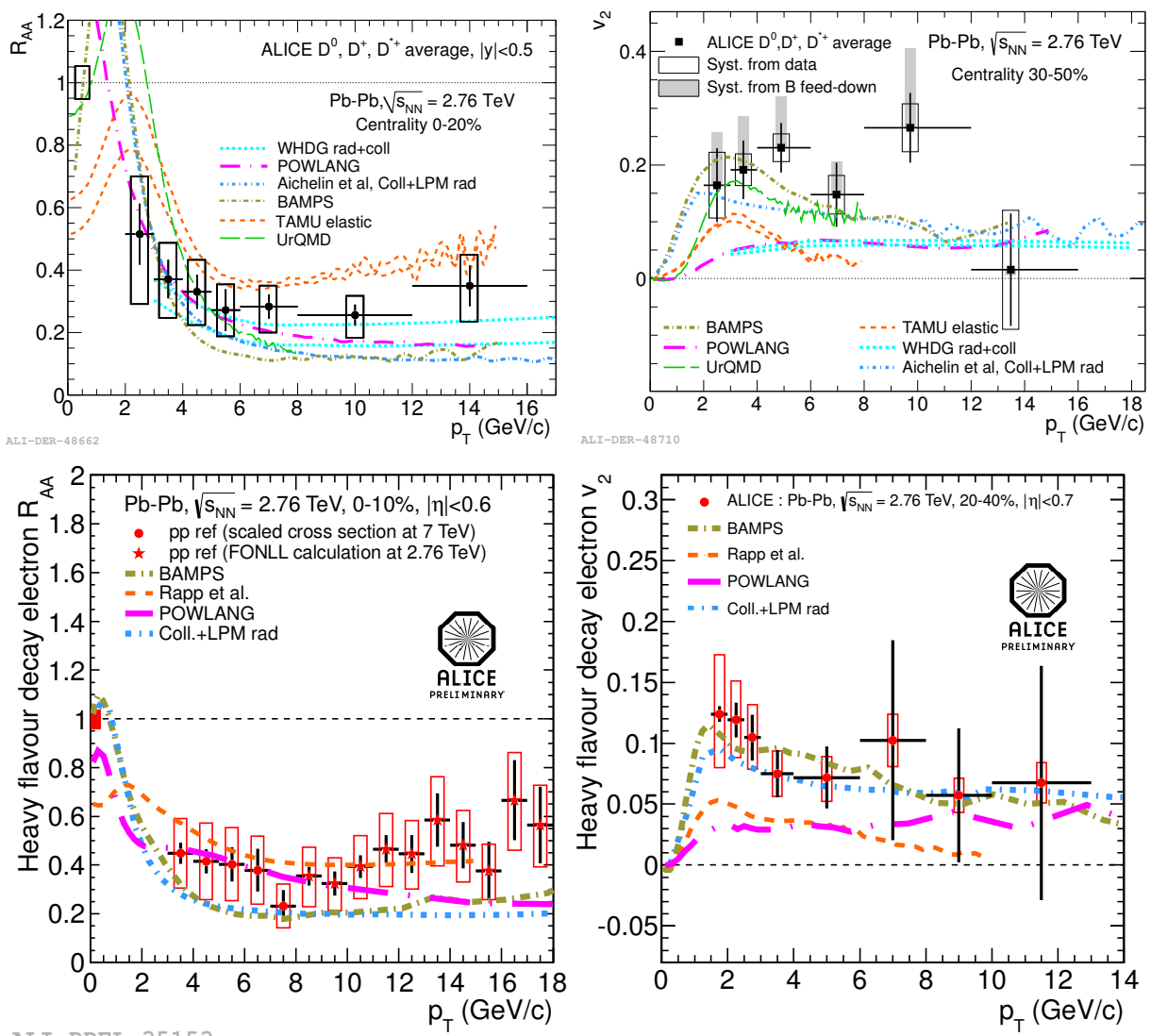

Figure 6. $R_{\mathrm{AA}}$ (left) and $v_{2}$ (right) of $\mathrm{D}$ mesons (top) and electrons from heavy-flavour hadron decay (bottom), compared to models: BAMPS [9], UrQMD [10], Coll+LPM rad [11], POWLANG [12], WHDG rad+coll [13], and TAMU elastic (Rapp et al.) [14].

[4] M. H. Thoma and M. Gyulassy, Nucl. Phys. B351, 491 (1991).

E. Braaten and M. H. Thoma, Phys. Rev. D44, 1298 (1991); Phys. Rev. D44, 2625 (1991).

[5] Y. L. Dokshitzer, D. E. Kharzeev, Phys. Lett. B519, 199 (2001).

N. Armesto, C. A. Salgado and U. A. Wiedemann, Phys. Rev. D69, 114003 (2004).

M. Djordjevic, M. Gyulassy, Nucl. Phys. A733 265 (2004).

B.-W. Zhang, E. Wang and X.-N. Wang, Phys. Rev. Lett. 93, 072301 (2004).

S. Wicks, W. Horowitz, M. Djordjevic and M. Gyulassy, Nucl. Phys. A783 , 493 (2007).

[6] N. Armesto, A. Dainese, C.A. Salgado and U.A. Wiedemann, Phys. Rev. D71, 054027 (2005).

[7] R. J. Glauber in Lectures in Theoretical Physics, NY, Vol. 1, 315 (1959).

M. Miller et al., Ann. Rev. Nucl. Part. Sci. 57, 205 (2007).

[8] J. Y. Ollitrault, Phys. Rev. D46, 229 (1992).

[9] O. Fochler, J. Uphoff, Z. Xu and C. Greiner, J. Phys G38, 124152 (2011).

[10] T. Lang, H. van Hees, J. Steinheimer and M. Bleicher, arXiv:1211.6912 [hep-ph].

T. Lang H. van Hees, J. Steinheimer, Y.-P. Yan and M.
Bleicher, arXiv:1212.0696 [hep-ph].

[11] P. B. Gossiaux, J. Aichelin and R. Bierkhandt, Phys. Rev C79, 044906 (2009).

P. B. Gossiaux, J. Aichelin, T. Gousset and V. Guiho, J. Phys G37, 094019 (2010).

[12] W. M. Alberico et al., Eur. Phys. J. C71, 1666 (2011). W. M. Alberico et al., J. Phys. G38, 164144 (2011).

[13] W. A. Horowitz and M. Gyulassy, J. Phys. G38, 124152 (2011).

[14] M. He, R. J. Fries and R. Rapp, Phys. Rev. C86, 014903 (2012).

[15] T. Matsui and H. Satz, Phys. Lett. B178, 416 (1986).

[16] S. Digal, P. Petreczky, H. Satz, Phys. Rev. D64, 0940150 (2001).

A. Mocsy, P. Petreczky, Phys. Rev. Lett. 99, 211602 (2007)

[17] B. Alessandro et al. (NA50 Collaboration), Eur. Phys. J. C39, 335 (2005).

[18] A. Adare et al. (PHENIX Collaboration), Phys. Rev. Lett. 98, 232301 (2007).

A. Adare et al. (PHENIX Collaboration), Phys. Rev. C84, 054912 (2011).

[19] P. Braun-Munzinger and J. Stachel, Phys. Lett. B490, 196 (2000).

[20] A. Andronic, P. Braun-Munzinger, K. Redlich, and J. Stachel, J. Phys. G38, 124081 (2011). 
[21] X. Zhao and R. Rapp, Nucl. Phys. A859, 114 (2011).

[22] Y.-P. Liu, Z. Qu, N. Xu, and P.-F. Zhuang, Phys.Lett. B678, 72 (2009).

[23] J. L. Albacete et al., Int. J. Mod. Phys. E Vol. 22 1330007 (2013), arXiv:1301.3395 [hep-ph].

[24] M. Cacciari, M. Greco and P. Nason, JHEP 9805, 007 (1998).

[25] M. Fasel, these proceedings.

[26] M. Cacciari, these proceedings.

[27] H. Woehri, these proceedings.

[28] B. Abelev et al. (ALICE Collaboration), Phys. Rev. Lett. 109, 072301 (2012).

[29] E. G. Ferreiro, arXiv:1210.3209 [hep-ph]; private communication.

[30] L. H. A. Manceau, these proceedings.

[31] G. Aad et al. (ATLAS Collaboration), Phys.Lett. B697, 294-312 (2011).

[32] S. Chatrchyan et al. (CMS Collaboration), JHEP 05, 063 (2012).

[33] CMS Collaboration, CMS PAS HIN-12-014 (2012).

[34] K. J. Eskola, H. Paukkunen and C. A. Salgado, JHEP 0904, 065 (2009).

[35] R. Vogt, Int. J. Mod. Phys. E Vol. 22, 1330007 (2013), arXiv:1301.3395 [hep-ph]; private communication.

[36] F. Arleo and S. Peign, arXiv:1212.0434 (2013). F. A. et al., arXiv:1304.0901 (2013).

[37] K. W. Hirotsugu Fujii, arXiv:1304.2221 (2013).
[38] E. Abbas et al. (ALICE Collaboration) arXiv:1303.5880 [nucl-ex].

[39] CMS Collaboration, CMS PAS HIN-12-007.

[40] R. Arnaldi for the ALICE Collaboration, arXiv:1211.2578 [nucl-ex].

[41] S. Chatrchyan et al. (CMS Collaboration), Phys. Rev. Lett. 109, 222301 (2012).

[42] B. Abelev et al. (ALICE Collaboration), JHEP 09, 112 (2012).

[43] Z. Conesa del Valle for the ALICE Collaboration, arXiv:1212.0385 [nucl-ex].

[44] C. Bianchin, these proceedings.

[45] B. Abelev et al. (ALICE Collaboration) Phys. Rev. Lett. 109, 112301 (2012).

[46] ATLAS Collaboration, ATLAS-CONF-2012-050 (2012).

[47] G. M. Innocenti for the ALICE Collaboration, arXiv:1210.6388 [nucl-ex].

[48] M. He, R. J. Fries, R. Rapp, Phys. Rev. Lett. 110, 112301 (2013).

[49] I. Kuznetsova and J. Rafelski, J. Phys. G32 S499S504 (2006).

[50] B. Abelev et al. (ALICE Collaboration), arXiv:1305.2707 [nucl-ex].

[51] A. M. Poskanzer and S. Voloshin, Phys.Rev. C58, 1671 (1998), arXiv:nucl-ex/9805001 [nucl-ex].

[52] B. Abelev et al. (ALICE Collaboration), arXiv:1205.5761 [nucl-ex]. 